\title{
THE INSTANTANEOUS POSITION AND DIAMETER OF SHORT DURATION BURSTS OF SOLAR RADIO EMISSION
}

\author{
J. A. HÖGBOM \\ Mullard Radio Astronomy Observatory, Cavendish Laboratory \\ Cambridge, England
}

It is known that the regions responsible for solar noise storms are associated with visible sunspot groups and that the diameter of such radio sources is not less than 7 minutes of arc at a wavelength of $3.7 \mathrm{~m}$. Little, however, is known of the size and location of the individual short-lived bursts (duration $\sim 1$ second). Two interferometric systems have been set up at $3.7 \mathrm{~m}$ to measure these quantities, and some preliminary results have been obtained:

(a) The diameters of single bursts. To measure the diameters of individual bursts an interferometer of spacing $270 \lambda$ was connected to three receivers. Receivers 2 and 3 gave records corresponding to total power interferometers in antiphase, while receiver 1 was arranged as a phase-switching interferometer in quadrature with the other two. From the relative sizes of an individual burst on the three records it is possible to determine the burst intensity, its angular size and position in one coordinate. Measurements carried out during a noise storm on 1958 March 21 gave an angular diameter of $6 \pm 1$ minutes of arc for about 25 individual bursts.

(b) The location of single bursts in two dimensions. In order to determine the instantaneous position of individual bursts, two phase-switching interferometers of spacing $135 \lambda$ were aligned in $\mathrm{E}-\mathrm{W}$ and $\mathrm{N}-\mathrm{S}$ directions. A third aerial was used with a total power receiver to measure the burst intensity. Preliminary results indicate that the positions of the individual bursts during a noise storm show a scatter of less than 3 minutes of arc.

Since the burst positions are spread over an area considerably smaller than that corresponding to the angular size of any individual burst, it seems likely that their large apparent size can be ascribed to a radiation scattering during its passage through the corona. 